\title{
Tango, Clubes e Inundación Análisis de la trama urbana desde sus crisis
}

\section{MARÍA EUGENIA ROSBOCH}

eugerosboch@gmail.com - Laboratorio de Investigación de Lazos Socio-Urbanos (LILSU), Universidad Nacional de La Plata (UNLP).

Fecha de recepción: 20 de abril de 2017

Fecha de aceptación: 19 de mayo de 2017

\section{RESUMEN}

En el presente artículo se hilvanan las investigaciones llevadas a cabo en los últimos diecisiete años de carrera como investigadora de la Universidad Nacional de La Plata. Período cuyo logro más significativo fue fundar en el año 2011, junto al equipo de investigación, el Laboratorio de Investigación de Lazos Socio-Urbanos (LILSU). El LILSU consolida una ardua trayectoria por materializar un campo de estudio, en ese momento erráticamente abordado por la comunicación, como es la compleja trama de los estudios en comunicación y ciudad.

Como hoja de ruta, se organizan cronológicamente los estudios emprendidos, comenzando con expresiones socioculturales como es la práctica del tango y la milonga, esta última como espacio de danza. Indagación que años más tarde nos conducirían a proponer el estudio de los clubes sociales y agrupaciones barriales, en tanto espacios de consolidación de lazos urbanos, para finalmente contemplar el análisis de los procesos de empoderamiento barriales que surgen frente a una inundación de características inusitadas, como fue la que se produjo el 2 de abril de 2013 en la ciudad de La Plata, la cual dejó a una comunidad sumergida en la miseria y el desencanto de sus mandatarios.

Todos estos temas suponen fenómenos muy disímiles, pero guardan relación no solo en los procesos teóricos y metodológicos comunicacionales con los que se emprende su análisis, sino también con un interrogante que permea las principales hipótesis de trabajo: ¿cómo se conforman y ac- cionan las redes sociales desde sus nudos más profundos, los lazos que se tejen en la comunidad barrial?

PALABRAS CLAVE: comunicación, ciudad, crisis, ciudadanía.

\section{ABSTRACT}

In the present article, I intend to link together all the inquiries I have carried out during the last 17 years as a researcher of La Plata University. In 2011 my enquiry team and I funded the Research Lab of Socio-Urban Bonding -LILSU (Laboratorio de Investigación de Lazos Socio-Urbanos) our most meaningful achievement. LILSU has consolidated a difficult path to make it real the research field of the complex correlation between communication and city, wrongly addressed by communication only. As a road map, I organize the studies undertaken chronologically, starting with sociocultural expressions such as the practice of tango and milonga, the last one as the place where people can dance. Years later, these studies would take me to pose the enquiry of the social clubs and neighborhood groups as places to strengthen urban bonding. Eventually these enquiriesled me to contemplate the analysis of the process of neighborhood empowerment arising from a flood of unusual characteristics, as it was the one that occurred on 2 April 2013 in La Plata city which left the community buried in misery and in complete disappointment of the government.

All these topics present different features but all of them are related to, not only in the theoretical and methodological communicational processes in which their analysis set off, but also with a question that passes through the main work hypothesis: how do social networks make up and act, from their deepest knots, all the bonding knitted in the neighborhood community?

KEYWORDS: communication, city, crisis, citizenship. 


\section{DESDE DÓNDE Y QUÉ INDAGAMOS}

En las páginas que aquí se presentan, se retoma y expone el trabajo de investigación realizado desde el año 2000 a la actualidad. Las investigaciones que se presentan son, en apariencia, variadas ya que implican objetos diversos que muestran procesos de construcción de sentido en prácticas que dialogan y proponen espacios muy distintos dentro del entorno urbano, pero que se unen en una impronta: la ciudad, y bajo una misma problemática, los lazos sociales perdidos, los recuperados y las nuevas formas de relación. En tal sentido, nos hemos detenido en el estudio de la milonga como espacio de danza del tango y la milonga como género musical; los clubes sociales como espacios de encuentro barrial; y las demandas sociales que surgen a raíz de la crisis societaria que produce la última gran inundación de la ciudad de La Plata, el 2 de abril de 2013.

Por lo expuesto, como hoja de ruta de las temáticas mencionadas, se considera necesario asumir un marco general desde donde concebimos los procesos de conformación y trasformación que atraviesan nuestras ciudades. Este marco se encuentra enraizado en situaciones de crisis; esto es, estimamos que son los momentos críticos los que nos permiten vivenciar con mayor exactitud y/o plenitud las rupturas y las continuidades en los imaginarios y procesos identitarios que se producen en la trama urbana.

Desde esa mirada de irrupción yerupción sociocultural proponemoscomo marco histórico de referencia dos momentos de crisis delos modelos de pensar y vivir la sociedad: la ruptura entre modernidad y posmodernidad —o segunda modernidad-, y el quiebre del modelo neoliberal que en la Argentina se materializó en el estallido social de 2001. Ambos procesos, aún inconclusos, conducen a investigar cómo eclosionan en el tejido social y, en consecuencia, qué tipo de vínculos conforman los ciudadanos en sus centros urbanos.

Laimportancia de tomar como punto de inflexión de nuestras investigaciones, nos condujo a observar que en las ciudades se yuxtaponen y/o entrecruzan dos estados de situación. Por un lado, están las crisis de carácter estructural antes mencionadas, pero también coexisten, por otra parte, las crisis coyunturales que impactan en la ciudad de tal forma que suponen un antes y un después en la vida cívica. Nos referimos a los fenómenos eco-ambientales que, dada la imprevisión delos sujetos, rápidamente se transforman en crisis político-sociales, como fue el caso antes mencionado de la inundación del 2 de abril de 2013 en la ciudad de La Plata, Argentina.

Incluir esos dos estados de situación nos orientó a formular dos dimensiones, solo discernibles en términos analíticos, delas acciones quecumplenla conformación de los imaginarios sociales a la hora de analizar las prácticas sociales. Una acción que interpreta específicamente las características estructurales y que abordamos desde las propuestas de Benedit Anderson (1993) y de Ernest 
Gelner (1995); y otra acción que indaga en la construcción imaginaria profunda, incluyendo su raíz emotiva, la propuesta por Armando Silva Téllez (2012).

Las perspectivas de análisis se abren de forma inusitada. Si bien esa situación es sumamente productiva para pensar y realizar investigaciones, también se corre el riesgo de perder la pertinencia y/o especificidad en nuestro estudio. Es por ello que a ese marco general es necesario sumarle nuestra perspectiva de análisis, poniendo de relevancia cómo consideramos la ciudad y los lazos identitarios que en ella se tejen y destejen. En ese sentido, consideramos que el espacio urbano le da forma y condiciona al agente que lo habita. A su vez, son sus pobladores quienes lo intervienen, crean y recrean. Es por ello que, en términos generales, rescatamos la postura de Jordi Borja (1998), quien propone estudiar a la ciudadanía desde sus prácticas, esto es, desde la apelación que la ciudad hace de ella:

La recreación del concepto de ciudadano, como sujeto de la política urbana, el cual se hace ciudadano interviniendo en la construcción y gestión dela ciudad. El marginal se integra, el usuario pasivo conquista derechos, el residente modela su entorno, todos adquieren autoes- tima y dignidad enfrentándose a los desafíos que les plantean las dinámicas y las políticasurbanas. El ciudadano es el que tiene derecho al conflicto urbano (1998, p. 49).

Tal perspectiva permite invertir el orden de la construcción del concepto de ciudadano y definirlo, no ya por sus orígenes, sino desde su emergente y su accionar en la trama urbana. Así, partir desde la visibilidad del proceso posibilita estudiar las manifestaciones de la acción ciudadana, articulando de forma inclusiva todas sus expresiones. Desde esa perspectiva es que asumimos que la ciudadanía como fenómeno identitario emergey/o se construye cuando la pertenencia a un espacio social se ve interpelada, ya sea por una situación de crisis que cuestiona derechos yobligaciones o por la celebración de su reivindicación.

La movilización de la ciudad nos conduce a pensar al habitante desde otras perspectivas, para ello recurrimos a Gravano (2008), quien analiza la participación ciudadana como modos de gestión social, esto es: "Como un proceso cultural, como forma de organizar y organizarse significativamente en la acción, en la cooperación social, en el más amplio de los sentidos" (2008, p. 10).

La noción de gestor, propuesta por Gravano, nos coloca frente a un ciudadano con poder de decisión y acción ante los problemas que atraviesa en su cotidianeidad. Visualizar ese empoderamiento es fundamental para poder analizar nuestras problemáticas a nivel barrial, haciendo especial énfasis en las acciones que llevan a cabo los miembros que la componen. Es así que nuestro recorte de estudio está dado por focalizar en las relaciones sociales que tejen la trama urbana desde sus expresiones cotidianas. Para ello nos situamos en el núcleo básico de construcción urbana: sus barrios, formaciones, organizaciones e instituciones comunitarias. 
Es en ese punto donde se observa claramente la impronta comunicacional de la mirada y el abordaje que hacemos de la ciudad. En este sentido, nos inscribimos en la línea propuesta por los estudios culturales, que abre la perspectiva de análisis focalizando la mirada en los procesos de conformación de sentido atravesados por relaciones de poder. Abanico de posibilidades que confluyen, desde múltiples pensadores, en una profunda discusión sobre el sistema social que nos condiciona; esa estructura que nosotros construimos a lo largo de nuestra historia y que tenemos las posibilidades de modificar. Conceptualizar la comunicación desde esa perspectiva encadena y le otorga coherencia a las posturas hasta aquí tomadas. Esto es, concebimos una sociedad que se organiza y moviliza en torno a tensiones y crisis más que al equilibrio auto-sustentado, que responsabiliza a los sujetos de sus acciones según sean los espacios sociales que ocupen en la estratificación cultural, que cuenta con las potencialidades necesarias para ser dueña de su destino.

Dentro de esa perspectiva, a su vez, nos focalizamos en la línea de análisis que propone la comunicación participativa ${ }^{1}$, ya que nos permite situarnos como parte interviniente de la investigación desdela acción conjunta con los sujetos investigados. Esto es, el analista es uno más en el campo de estudio, quien, desde la realización de un diálogo conjunto, construye elementos para la acción ciudadana, ya sea en el descubrimiento de sus potencialidades como en la materialización de prácticas concretas que le otorguen un cambio en su calidad de vida.

\section{DANZA URBANA: EL TANGOYLA MILONGA}

Como se aclara al inicio de este trabajo, la investigación que orienta la mirada analítica acerca de los lazos sociales que conforman la ciudad, fue elaborada en el marco del estudio sobre las danzas del tango en las milongas, entendidas como los espacios de producción del tango. Para poder arribar a un análisis pormenorizado de los imaginarios sociales del tango, se diferenciaron dos espacios de producción simbólica: por un lado, aquellos lugares donde se reproduce un tango de espectáculo, es decir, el preparado para los centros de consumo cultural, tanto nacionales como trasnacionales; y, por el otro, el tango que se crea y recrea en las milongas entendidas como espacios de interacción social que originan, producen y reproducen el tango.

Para desentrañar los sentidos que inviste la milonga como espacio de representación del tango y evaluar la relación que éste guarda con los imaginarios surgidos de los espacios del espectáculo, se consideró necesaria la realización de un análisis procesual, estableciendo que el tango -como

1 El concepto de comunicación participativa encuentra sus antecedentes en Latinoamérica en procesos de significación que tiene como principales referentes a Mario Kaplum (2002), en materia de Comunicación y Educación, y a Jesús Martín Barbero (1987), en torno a sus aportes sobre comunicación popular. 
práctica cultural que remonta sus orígenes a mediados del siglo XIX-atraviesa cuatro períodos históricos:

- Embrionarioy conformación(1850-1920): abarca entre 1850a 1920, donde el negro, gaucho e inmigrante europeo plasman el tango.

- Popularización (1920-1966): Delimitación que toma en cuenta la incorporación de la clase obrera y comerciante, así como de las élites dominantes (nacionales y transnacionales), al tango. Se enmarca en las décadas que transcurren entre 1920 y 1966, cuando el tango se massmediatiza y se exporta a los circuitos de consumo en el exterior, principalmente por la actividad de los emergentes maestros de danza.

- Descorporización (1966-1983): está signado por los sucesivos gobiernos militares que se vivieron en Argentina desde 1966 a 1983. Es denominado así ya que es un momento que, dadas las políticas represoras aplicadas, se desvincula al tango de sus agentes de producción, es decir, de los sectores marginales (vínculo que ya no se restablecerá) y de los sectores medios (obreros y comerciantes) al borrarse a la milonga del espacio público efecto de una imposición política autoritaria que condujo al repliegue de la sociedad argentina al ámbito de lo privado.

- Resurgimiento (1983 a la actualidad): comienza a desarrollarse en 1983 con el retorno de la democracia, lo cual generó la reorganización de las milongas. Este 'resurgimiento' es un proceso que continúa hasta la actualidad.

Asimismo, es oportuno aclarar que esos períodos históricos se imbrican con dos momentos paradigmáticos de la acción social (Turner, 1974): el proceso de formación del Estado argentino (1853/1890) y la transición democrática (1983/ hasta la actualidad).

La diferencia está en que esos momentos paradigmáticos constituyen eventos críticos de la acción social que, en tanto tales, orientan la incorporación práctica delos múltiples sentidos que se expresan en los espacios de representación del tango. Es decir, traslapan el proceso de conformación y transformación del tango, se enraíza y, en consecuencia, condicionan las representaciones que configuran al tango como práctica cultural.

El establecimiento de esos períodos, y sus raíces en profundos procesos históricos que transformaron la vida cívica del país, permitió indagar la conformación, resquebrajamiento y revitalización de los lazos sociales que se producen en nuestros espacios urbanos, así como observar cambios en la construcción de vínculos identitarios que van migrando de espacios y constitución. Preocupa-

2 Los períodos históricos se establecieron siguiendo la categoría de tradición selectiva de Raymond Williams (2000), mediante la cual el autor establece que los órdenes hegemónicos se conforman y trasforman mediante procesos de construcción simbólica de características dominantes, emergentes y residuales donde los dos últimos tienden a cuestionar al primero. 
ción académica que creció y terminó materializándose en otras investigaciones dedicadas a pensar, por ejemplo, los clubes sociales como espacios legitimados en el período de popularización del tango, los cuales se descorporizan perdiendosus vínculos sociales de referencia, formando, con todo, parte de una esquiva mirada nostálgica que se niega a ver el estado actual de esas antaño pujantes instituciones.

\section{LOS CLUBES DE ANTES, LOS CLUBES DE HOY}

La huella que dejan los clubes sociales en sus momentos de esplendor en la memoria urbana se puede rastrear desde diversos lugares. Las narraciones de quienes vivenciaron ese momento y añoran revivirlo, los que crecieron entre relatos devenidos en fábulas de tíos, padres y abuelos, hasta la eterna barra de la esquina que vio el club vaciado, algo olvidado y que busca su reencuentro. Por tanto, como una primera síntesis, asumimos a los clubes sociales como espacios barriales instituidos de encuentro sociocultural que, al propiciar la participación, crean y refuerzan vínculos urbanos indispensables para la generación de ciudadanía.

Desde esas inquietudes, es que, en un primer momento, nos propusimos estudiar el proceso de gestación y actual significación de los clubes sociales de la ciudad de La Plata, con el fin de establecer cuáles potencialidades invisten como espacios de reconstrucción y consolidación de vínculos urbanos ${ }^{3}$. En el trabajo de campo preliminar, comprendimos que nuestro objeto de estudio se tornaba primordial a los fines que nos alentaban, ya que los clubes sociales, en tanto formaciones intersticiales alternativas a espacios instituidos, desde su fundación se instauran como espacios barriales y comunitarios que crean sólidos vínculos vecinales.

Un punto crítico en la historia de los clubes y sus lazos identitarios con el barrio, a nuestro entender, se produce por las últimas dictaduras militares. Esto es, consideramos que los vínculos entre el club y el barrio de referencia son prácticamente desmantelados por las prácticas represivas implementadas por los gobiernos de facto que provocan el repliegue de la sociedad al ámbito de lo privado, situación que se profundiza con la implementación en la década de 1990 de políticas neoliberales que promueven al individualismo y la separatividad. En otras palabras, el análisis de los clubes nos permite comprender la desarticulación sociocultural que sufrió nuestro país en los negros años dictatoriales y los narcotizantes años noventa argentinos.

Dada la historia de nuestros clubes sociales, se nos presentan como espacios que, desde su nacimiento, invisten la capacidad de generar diálogos

3 Proyecto de investigación del Programa de Incentivos Decentes de Investigación de la UNLP, P/140: “Los clubes sociales: hangares vacíos o potenciales espacios de reconstrucción y consolidación de vínculos urbanos”. Directores: María Eugenia Rosboch y Flavio Peresson. Integrantes: María Ofelia Tellechea, María Mónica Malagamba, Virginia Cáneva y Hernán Mendoza Jaufret. 
transgeneracionales e interculturales, promover algún tipo de apropiación identitaria o gestar fuertes compromisos cívico-político, atributos hoy dormidos que consideramos indispensable despertarlos para comenzar a regenerar el tejido social rasgado por los procesos históricos mencionados. Para enfrentar este reto, nos propusimos como objetivos generales establecer cuáles son las características históricas de los clubes de barrio de la ciudad de La Plata que aún perduran, y cuáles de esas características cambiaron en relación al proceso de gestación y actual transformación de sentidos. Dicha caracterización nos permitió, por otra parte, determinar y pensar las potencialidades que invisten la reconstrucción y consolidación de vínculos urbanos y revitalizar el papel que cumplieron y podrían cumplir los clubes sociales en la construcción de vínculos urbanos de la ciudad de La Plata, ya que son formaciones intersticiales alternativas a otros espacios instituidos y encierran un papel central en el marco de las organizaciones barriales. Propósitos de diferente envergadura que pudimos desarrollar a partir de trabajos de extensión que posibilitaron la intervención en distintos clubes platences y de la provincia de Buenos Aires.

A los fines de este artículo, es importante resaltar que la pregunta que impulsó, de acuerdo a nuestras perspectivas de análisis, el estudio de los clubes sociales radicó principalmente en la indagación de los espacios urbanos que ocuparon el lugar cedido o perdido por los clubes, interrogante que a su vez contempla la necesidad de observar la relación que esos clubes tienen con los nuevos espacios de interacción social que surgen en los barrios de La Plata. Dicho interés se materializó finalmente en dos proyectos de investigación denominados "Del ostracismo social al compromiso colectivo. Configuración y reconfiguracion de espacios urbanos de participación ciudadana" y "Citas urbanas: construcción y regeneración de lazos socioculturales en la ciudad”. Este último, reorientado a la problemática hídrica frente a los trágicos hechos producidos por la inundación del 2 de abril de 2013.

\section{LA INUNDACIÓN. ¿CÓMO REACCIONAR FRENTE A LA CRISIS?}

La inundación de año 2013 que asoló a la ciudad de La Plata, supusola generación de acuerdos entre los distintos organismos del Estado y la Universidad para que la comunidad académica trabajara en conjunto sobre la problemática hídrica de la región. Es así que las investigaciones que abordamos sobre dicha problemática son parte de una serie de estudios que se encuentran articulados en tres proyectos de investigación: "La ciudad y sus transformaciones. Estudio sobrela acción ciudadana en momentos de crisis eco-ambientales y/o políticosociales", concluido en diciembre de 2015 y continuado por otro estudio aún vigente titulado: "Diálogos urbanos. Intervenciones en el medioambiente desde la acción y gestión ciudadana”. A ambos proyectos, aceptados en el marco 
del Programa de Incentivos a la Investigación de la Universidad Nacional de La Plata (UNLP), se le agregó el estudio denominado "Construcción de un sistema integrado de gestión del riesgo hídrico en la Región del Gran La Plata", el cual fue dirigido por Jorge Karol en el marco de un convenio finalizado en agosto de 2016 entre el Consejo Nacional de Investigación Científica y Tecnológica (CONICET) y la UNLP4

De lo expuesto, consideramos particularmente fructífero el último proyecto mencionado, ya que implicó una iniciativa interdisciplinaria que, por primera vez, puso en diálogo a distintos saberes del campo académico y a instituciones que se unieron para intercambiar recursos humanos y económicos. Si bien al interior del grupo hubo momentos en que los encuentros fueron signados por la incomprensión, las vallas disciplinarias no fueron obstáculos para arribar a acuerdos conceptuales y metodológicos que hicieron del proceso una experiencia enriquecedora.

En ese marco, el LILSU fue convocado para trabajar sobre el área de comunicación. Es pertinente aclarar que desde nuestros parámetros pusimos el sesgo en trabajar la comunicación desde una perspectiva que se aleja de las clásicas miradas que restringen a la disciplina al campo de los medios de difusión masiva y/o a la labor periodística. Si bien incorporamos esos saberes, nuestra mirada es más amplia y nos propusimos trabajar con las comunidades afectadas por la inundación desde sus redes básicas, hasta las más complejas, esto es, desde el barrio y sus organizaciones hasta el diálogo que éstos puedan o no establecer con los decidores políticos, ya sean municipales como provinciales y/o nacionales.

La descripta situación muestra la necesidad de realizar trabajos que releven los vínculos espontáneos de relación social y que indaguen sobre los sistemas de relación entre la comunidad afectada y los estamentos de gobierno, no solo diagnosticando su estado, sino también evaluando e incentivando canales autogestionados de diálogo que permitan la articulación de protocolos de prevención y mitigación del riesgo por inundación.

Frente a lo expuesto, como aportes desde nuestro laboratorio a la problemática hídrica de la región, se relevaron las redes sociales virtuales vinculadas a la inundación y las agrupaciones de vecinos auto-convocados ${ }^{5}$ que surgieron después de la inundación. También se seleccionaron las zonas poblacionales donde se realizó el trabajo de campo y se mapearon los organismos guberna-

4 Coordinador General. MSc. Soc. Jorge Karol (Facultad de Arquitectura y Urbanismo, UNLP). Equipo responsable de la coordinación por áreas (en orden alfabético): Dr. Daniel Del Cogliano (Facultad de Ciencias Astronómicas y Geofísicas, UNLP), Dra. Graciela Etchegoyen (Facultad de Ciencias Médicas, UNLP), Ing. Fernanda Gaspari (Facultad de Ciencias Agrarias y Forestales, UNLP), Dr. Eduardo Kruse (Facultad de Ciencias Naturales y Museo, UNLP), Dr. Marcelo Naiouf (Facultad de Informática UNLP), MSc. Ing. Pablo Romanazzi (Facultad de Ingeniería, UNLP), Dra. Eugenia Rosboch (Facultad de Periodismo y Comunicación Social, UNLP), Dr. Arq. Gustavo San Juan (Facultad de Arquitectura y Urbanismo, UNLP); Dr. Ramiro Sarandón (Facultad de Ciencias Naturales y Museo, UNLP).

5 Véase el trabajo realizado por la Dra. Virginia Cáneva (2016), una referente en la investigación original sobre agrupaciones autoconvocadas no partidario-gubenamentales. 
mentales involucrados en la problemática hídrica de la ciudad de La Plata, Berisso y Ensenada -este último trabajo fue dirigido y ejecutado por Jorge Karol-. Paralelamente, se identificaron las organizaciones autoconvocadas vinculadas a problemáticas medioambientales que exceden los hechos producidos por la inundación. En términos generales, se observó que la problemática medioambiental, si bien se reinstaló fuertemente en la región por motivos de la última inundación, no es una temática prioritaria en la agenda ciudadana, tampoco la experiencia vivida generó niveles de responsabilidad en la población en general sobre su intervención en el ecosistema.

\section{OBSERVACIONES FINALES}

A modo de cierre, consideramos necesario enfatizar que el camino recorrido se realizó siguiendo parámetros metodológicos que inscriben a las investigaciones antes mencionadas en un paradigma de corte cualitativo en la recolección e interpretación de datos. Los cuales, a su vez, estuvieron sustentados en talleres y seminarios donde nos propusimos la intervención de los investigadores en la problemática analizada.

Es también necesario aclarar que la misma no consistió en la mirada altiva del erudito que se dispone a aconsejar o asesorar a la comunidad. Por el contrario, la relación que se estableció es de carácter pedagógico, donde todos los interlocutores intercambiamos saberes asumiendo las responsabilidades que cada uno ocupa en la trama de la acción. Es en ese sentido que consideramos que nuestro trabajo se enmarca en el campo de la comunicación participativa. 


\section{REFERENCIAS}

Anderson, B. (1993). Comunidades imaginadas. Reflexiones sobre el origen y la difusión del nacionalismo. México: Fondo de Cultura Económica.

Barbero,J.M.(1987).Delosmediosalasmediaciones. Comunicación, culturayhegemonía. México: Gilli.

Borja,J.(1998). Ciudadanía y espacio público.En PepSubirós(ed.). Ciudad Real, Ciudad Ideal. Significado y función en el espacio urbano moderno. Barcelona: Centro de Cultura Contemporánea de Barcelona.

Caneva, V. (2016). Crisis y encuentros: una mirada comunicacional sobre la recreación de lazossocio-urbanosenorganizacionesdevecinosautoconvocados. Tesisdedoctorado delDoctorado en Comunicación, Facultad dePeriodismo y Comunicación Social de la Universidad Nacional de La Plata (UNLP). En línea: http://sedici.unlp.edu. ar/handle/10915/51386

Gelner, E. (1995). Antropología y Política: revolución en el bosque sagrado. Barcelona: Gedisa.

Gravano, A. (2008). Imaginarios barriales y gestión social. En IX Congreso Argentino de Antropología Social. Facultad de Humanidades y Ciencias Sociales, Universidad Nacional de Misiones (UNM), Argentina.

Kaplun M, (2002). Una pedagogía de la comunicación (el comunicador popular). La Habana: Caminos.

Rosboch, M. E. (2006). La rebelión de los abrazos. Tango, milonga y danza. Imaginarios del tango es sus espacios de construcción simbólica: la milonga y el espectáculo. La Plata, Argentina: Ed. Universidad Nacional de La Plata (UNLP).

Rosboch, M. E. et al. (2006). Los clubes sociales. Hangares vacíos o potenciales espacios de construcción ciudadana. En revista Oficios Terrestres, 18(12), Facultad de Periodismo y Comunicación Social (FPyCS), Universidad Nacional de La Plata (UNLP), Argentina, pp. 82-89.

Rosboch, M.E. (2017). Imaginarios en acción. Reclamos y reivindicaciones ciudadanas ante la inundación. En Imagonautas. Revista Interdisciplinaria sobre Imaginarios Sociales, No 9, Universidad de Vigo, Pontevedra, España, pp. 36-53.

Silva Téllez, A. (2012). Álbum de Familia. La imagen de nosotros mismos. Colombia: Universidad de Medellín.

Williams, R. (2000). Marxismo y Literatura. Barcelona: Península/Biblos. 


\section{IDENTIFICACIÓN DE LAAUTORA}

María Eugenia Rosboch es Doctora en Antropología por el Centro de Investigación y Estudios Superiores de Antropología Social (CIESAS), estudios de posgrado reconocidos por el Consejo Nacional de Ciencia y Tecnología (CONACYT), México. Es Magister en Comunicación por la Universidad Iberoamericana (UIA), México, y Licenciada en Comunicación Social por la Facultad de Periodismo y Comunicación Social (FPyCS) por la Universidad Nacional de La Plata (UNLP). Es Profesora e Investigadora categoría II del Programa de Incentivos a la Investigación y la Docencia de la FPyCS. Actualmente, dirige el Laboratorio de Investigación de Lazos Socio-Urbanos (LILSU), FPyCS/UNLP. Fue directora y creadora de la Estancia Posdoctoral en Comunicación, Medios y Cultura de la FPyCS/ UNLP. Ha publicado diversos libros y artículos sobre temas vinculados a la línea de investigación que desarrolla en comunicación, cultura y ciudad. Es miembro dela Red de Investigadores en Imaginarios y Representaciones (RIIR).

\section{REGISTRO BIBLIOGRÁFICO}

Rosboch, María Eugenia (2017). Tango, Clubes e Inundación. Análisis de la trama urbana desde sus crisis. InMediaciones de la Comunicación, 12(1), 179-189. 